\title{
PRÉSENTATION
}

Les animaux, leur industrie et leurs mœurs, constituent un des thèmes les plus constants de la vulgarisation scientifique depuis deux siècles '. Du Spectacle de la nature de l'abbé Pluche jusqu'au cinéma animalier, une abondante production a été consacrée à ce sujet. Son but premier est certes la diffusion d'un savoir zoologique. Ce savoir est, dans une grande mesure, complémentaire de celui donné par l'école. En effet, l'enseignement des "sciences naturelles" dominé autrefois par l'anatomie, aujourd'hui par la physiologie et la biologie moléculaire, laisse peu de place à la description des comportements, qui constitue en revanche l'objet quasi exclusif de la vulgarisation dans ce domaine. Cette zoologie vulgarisée est au demeurant très sélective. Elle a ses vedettes, parfois détrônées, mais aussi ses éternels oubliés; le Lion y apparait plus fréquemment que la Belette. Elle a ses sujets de prédilection et ses zones d'ombre; les noces cruelles de certains Arthropodes sont plus souvent évoquées que le régime alimentaire du Lapin de garenne. Malgré ces disparités, elle participe à une circulation de connaissances dont il serait intéressant de mesurer l'impact en n'oubliant pas que pour une partie du public, elle rencontre un savoir naturaliste antérieur, de nature empirique, sans lequel certaines activités de chasse, de pêche ou d'élevage - de même que certaines attitudes à l'égard de la protection de l'environnement - seraient tout simplement impossibles.

\section{L'HOMME ET L'ANIMAL}

Derrière cette fonction didactique, qui distingue la vulgarisation zoologique d'autres types de discours sur l'animal, se profile une autre fonction souvent implicite mais qui est parfois avouée sans détour: éclairer

1. Je remercie pour leurs remarques et leurs suggestions sur ce texte Bernadette Bensaude et Jorge Martinez.

Revue de synthèse : IV S. Nos 3-4, juil.-déc. 1992. 
l'homme sur lui-même. Cette opération comporte deux faces. Elle consiste d'abord à souligner tout ce qui relève de l'animal dans l'homme. Prise à la lettre, l'humanité est logiquement incluse dans l'animalité comme l'un de ses genres, et c'est le terme de "bestialité » qui devrait s'employer ici, s'il n'était pas trop fortement connoté. Au demeurant, l'acception scolastique de la notion d'animal comme "ètre animé ", comprenant à la fois la "bête " et l'« homme ", est aujourd'hui obsolète et le terme d'animalité traduit assez bien l'idée du caractère animal au sens de non-humain. Présenter des activités humaines comme la répétition, un peu plus élaborée, de comportements animaux est un motif récurrent de la vulgarisation scientifique. Son objectif avoué est généralement de donner à l'homme une leçon d'humilité. Seul un anthropocentrisme naîf nous empêcherait de percevoir dans nos arts, nos amours, nos sociétés et nos guerres, une simple version - grossie par la proximité des comportements animaux les plus courants. Ce thème n'appartient pas en propre à la vulgarisation, en quelque sens qu'on entende ce terme ; il lui est bien antérieur; en témoignent, par exemple, ces pages de l'Apologie de Raymond Sebond où Montaigne rassemble une multitude d'anecdotes sur la moralité et l'intelligence des bêtes, raille l'obstination des hommes à se considérer comme supérieurs, et conclut :

" Par où il appert que ce n'est pas vray discours, mais par une fierté folle et opiniatreté, que nous nous préférons aux autres animaux et nous séquestrons de leur condition et société ${ }^{2}$.

Cependant comme le notait Georges Canguilhem en 1960, précisément à propos de Montaigne, "l'anthropocentrisme est plus aisé à rejeter que l'anthropomorphisme ${ }^{3}$. On pourrait même avancer que la critique de l'anthropocentrisme, dans ce contexte, se fonde presque toujours sur un anthropomorphisme préalable. C'est sans doute parce que les comportements animaux sont conçus sur le modèle de nos activités que nous pouvons avec tant de facilité y retrouver l'équivalent de ces dernières. Mais si l'animal ne fait que rendre au vulgarisateur ce que celui-ci lui a prêté, il faut reconnaître qu'il le lui rend au centuple. L'anthropomorphisme, toujours décrié, est souvent inévitable. Comment décrire la fuite d'une proie sans évoquer sa peur, la stratégie d'un prédateur sans parler de ruse, la recherche d'un partenaire sans prononcer le mot de

2. Montaigne, Essais, liv. II, chap. XII, Paris, Gamier, 1962, p. 496-537, citation p. 537.

3. Georges Cangulliem, « L'homme et l'animal du point de vue psychologique selon Charles Darwin ", 1960, in ID., Etudes d'histoire et de philosophie des sciences, Paris, Vrin, 1983 , p. 121. 
séduction? Les critiques contre l'anthropomorphisme ne sont d'ailleurs pas sans ambiguité. Le terme lui-même est d'emploi relativement récent dans ce sens. Littré qui ne lui consacre que deux lignes le cantonne dans son sens théologique: "Doctrine de ceux qui attribuent à Dieu une forme humaine. " Autrefois méconnaissance de la transcendance divine, l'anthropomorphisme semble avoir gardé de ses origines religieuses, un relent de sacrilège. Aujourd'hui, puisque son caractère peccamineux n'est plus de rabaisser la divinité en lui donnant forme humaine, lui reprochet-on de rabaisser la forme humaine en la prêtant à l'animal non humain, ou de réduire la diversité du vivant en gommant des différences?

Les contributions rassemblées ici invitent à poser ces questions en même temps qu'elles en soulèvent beaucoup d'autres. En particulier, celle de savoir quel type d'animal est associé à un type de discours. Si les insectes offrent un thèrne de choix pour la vulgarisation c'est, d'une certaine manière, parce qu'ils obtiennent des effets proches de ceux de l'activité humaine par des moyens complètement différents. À cet égard, les singes se situent à l'opposé des insectes. Proches des hommes par l'aspect extérieur et par leurs capacités individuelles, ils surprennent et fascinent par la simplicité apparente de leur vie sociale, tout en irritant aussi comme des caricatures de l'humain. De tels problèmes semblent traverser les périodes et revenir de manière récurrente d'une époque à l'autre. Une telle approche encourt fatalement le reproche d'anachronisme. On objectera, par exemple, que le changement dans la sensibilité à l'égard des animaux n'a pu qu'affecter le statut symbolique de l'animalité. En fait, on peut très bien admettre l'existence de phénomènes de longue durée dans le rapport symbolique de l'homme à l'animal et, cependant, chercher à dégager les changements dans ce domaine et à repérer les discontinuités ${ }^{4}$.

On peut considérer, au moins comme hypothèse de travail, que l'animalisation des conduites humaines et la description anthropomorphique des comportements animaux sont des attitudes récurrentes qu'on retrouve, avec des modalités et des significations différentes, tout au long de la période qui va du début du Xvir' siècle jusqu'à aujourd'hui. La question des limites temporelles se pose peut-être avec plus d'acuité à propos de la vulgarisation elle-même.

4. Cf. Keith Thомas, Dans le jardin de la nature. La mutation des sensibilités à l'époque moderne, éd. orig. 1983, trad. de l'anglais par Catherine MaLAmoud, Paris, Gallimard, 1985. Récemment est parue une livraison de la revue Alliage intitulé "L'animal. L'homme ", 7-8, printemps-été 1991, qu'on consultera avec profit, ainsi qu'un article de François Sigaur, "L'animal machine ou personne? ", Ethnozootechnie, 46, mars 1991, qui montre comment une éthologie scientifique n'a pu se construire qu'en se libérant de la conception cartésienne et des postulats behavioristes. 
DIFFUSION, POPULARISATION, VULGARISATION

À partir de quand peut-on parler de vulgarisation? Plusieurs dates ont été proposées.

Bruno Béguet, dans l'ouvrage très documenté, publié récemment par la bibliothèque $\mathrm{du}$ Conservatoire national des arts et métiers sous sa direction, date "l'invention " de la vulgarisation du début des années 1850. Cette " invention " résulterait, selon lui, de la conjonction de plusieurs faits : un public large et bien disposé, une communauté scientifique reconnue et consciente d'elle-même, une presse et une édition en pleine expansion $^{5}$. Il est de fait que l'augmentation du nombre des publications et la professionnalisation de la fonction - le tout lié au développement de la presse, aux succès des expositions universelles, aux progrès de la scolarisation - créent une situation nouvelle et semblent marquer l'apparition de la vulgarisation, si on définit celle-ci selon des critères uniquement sociologiques ${ }^{6}$. Il y a incontestablement un effet de masse qui fait du milieu du siècle un tournant décisif. Un tournant mais non un point de départ. Il apparaît, en effet, que les thèmes développés dans la deuxième moitié du siècle étaient déjà formulés dans les décennies précédentes. Ainsi, l'auteur du Dictionnaire pittoresque d'histoire naturelle et des phénomènes de la nature explique dans l'Introduction, parue en 1834, qu'il entend " faire participer les masses aux belles découvertes que les savants ont faites dans le domaine des sciences naturelles $»$. Pour se défendre de faire double emploi avec les dictionnaires existants, il précise que

« [ces dictionnaires sont] remplis des mots et des définitions les plus élevées de la science, et par cela même hors de portée des gens du monde et des étudiants qui ne tiennent pas à savoir, par exemple, si tel ou tel auteur a divisé le genre mouche, que tout le monde connait, en 2000 petits groupes, basés sur la présence d'un poil de plus ou de moins aux mâchoires, mais qui apprendraient avec intérêt, au contraire, comment les mouches se multiplient, quelles sont leurs habitudes, les ruses qu'elles emploient pour se

5. La Science pour tous. 1850-1914, dir. Bruno Béguet, Paris, Bibliothèque du C.N.A.M., 1990, p. 6-7. Depuis la rédaction du présent article est paru un autre ouvrage, également stimulant et riche en informations, celui de Daniel RaICHvarg et Jean JacQues, Savants et ignorants. Une histoire de la vulgarisation scientifique, Paris, Seuil ("Science ouverte "), 1991. Tout en montrant la spécificité de la vulgarisation du xxe siècle (« l'âge d'or " de cette littérature), les auteurs ne négligent pas pour autant ses antécédents.

6. Indépendamment de ces dónnées sociologiques, Bruno Béguet considére que "l'état "préscientifique " du xvir siècle, pendant lequel règnent cet " esprit concret " et cette " âme puérile et mondaine " évoqués par Bachelard dans la Formation de l'esprit scientifique, rend très aléatoire la recherche d'antécédents exacts à la vulgarisation ". 
soustraire à leurs ennemis, les moyens que nous avons de les détruire, etc., etc. Un dictionnaire spécialement destiné à faire connaître l'histoire naturelle sous ce point de vue deviendrait bientôt populaire $[\ldots]{ }^{7}$.

Il est certain qu'un tel texte reflète la cassure qui s'est créée au tournant du XviII et du XIX ${ }^{\mathrm{e}}$ siècle entre, d'une part, les soucis des naturalistes, professionnels ou amateurs motivés, soucieux avant tout de précision et de rigueur dans la description anatomique et la classification, et, d'autre part, les intérêts du public pour une histoire naturelle colorée et narrative, qui fasse rêver tout en se montrant utile. Mais on trouverait des textes analogues au cours du XviI' ${ }^{e}$ siècle. Ainsi, un certain Bazin, auteur, en 1747, d'une histoire des insectes qui n'est de son propre aveu qu'un abrégé des Mémoires de Réaumur, justifie son projet en ces termes :

" Les Scavans qui font des découvertes, traitent en Scavans : ils s'étendent, pèsent sur les circonstances qui sont importantes entre eux et pour les progrès des Sciences, mais souvent indifférentes et quelquefois rebutantes pour la partie du Public qui est plus curieuse de connoître les découvertes que la manière dont elles ont été faites ${ }^{8}$.

La reconnaissance de cette continuité de projet permet d'éviter les difficultés qu'entraine une définition trop étroite de la vulgarisation. Comment, en effet, pourrait-on ne reconnaître comme vulgarisateurs que les auteurs qui se présentent sous ce label, puisqu'à l'intérieur même de la période 1850-1914, un des maîtres du genre, Camille Flammarion, critique le terme de "vulgarisation »; il entend, pour sa part, " populariser » et non « vulgariser » la science ${ }^{9}$.

Le verbe populariser et le substantif popularisation, apparus bien avant vulgariser et vulgarisation, continuent à être employés tout au long du siècle. Ils peuvent, comme chez Flammarion - ou chez Raspail - , servir à marquer une volonté de rendre la science populaire emportant par-là une critique implicite ou explicite à l'égard d'une science considérée comme établie, académique voire officielle, et s'accompagnant en même temps de l'idée qu'on peut demander un effort au lecteur du moment

7. Dictionnaire pittoresque d'histoire naturelle et des phénomènes de la nature, dir. F.-E. Gutrin, Paris, 1834-1840, t. 1, introduction, p. v-viu.

8. BAzIN, Abrégé de l'histoire des insectes..., cité in Jean Lhoste, Les Entomologistes français, 1750-1950, s. 1., I.N.R.A.-O.P.I.E., 1987, p. 37.

9. « Nous voulons populariser la science, c'est-à-dire la rendre accessible [...], mais nous ne voulons pas la vulgariser [...] " Cf. Camille Flammarion, L'Astronomie, $1^{\text {re }}$ année, 1883, p. 3, n. 3, cité par Bernadette Bensaude, "Camille Flammarion et la science populaire ", "Sciences pour tous ", Romantisme, 65, 1989, p. 103. L'ensemble des contributions rassemblées dans cette livraison de Romantisme apporte des analyses et des références sur la vulgarisation au $\mathrm{xIX}^{e}$ siècle. 
mais qu'on ne lui suppose pas de connaissances préalables particulières ${ }^{10}$. À l'inverse, la formule les gens $d u$ monde, par laquelle beaucoup d'ouvrages désignent encore leur public supposé, évoque une société de lecteurs cultivés mais à qui il serait déplacé de demander trop d'attention. Il convient cependant de ne pas accorder ici trop de poids à ces questions de terminologie : ainsi, la traduction française du livre de Schleiden sur La Plante et la vie, porte en sous-titre : « leçons populaires de botanique à l'usage des gens du monde " ".

Plus que par leur public supposé, les projets de diffusion des connaissances se distinguent par leur finalité. À travers les styles et les époques, trois types de fins reviennent avec une grande constance et définissent par leur combinaison ou par la prédominance de l'une d'elles, le projet de chaque auteur. La première est évidemment de satisfaire la curiosité. La deuxième de fournir des connaissances utiles. La troisième de tirer une leçon philosophique ou religieuse des connaissances scientifiques ${ }^{12}$.

Définir la vulgarisation par ses finalités amène nécessairement à la replacer dans la longue durée comme un phénomène qui accompagne la science moderne tout au long de son histoire. Dès lors que des milieux scientifiques - fussent-ils numériquement faibles - commencent à se constituer avec leurs codes, leurs règles, leurs moyens de communication, on voit apparaître des auteurs qui cherchent à transmettre, à un public non captif (par opposition au public scolaire) et situé hors des milieux scientifiques, une partie de la production intellectuelle de ces milieux. Ces auteurs, tantôt ont déjà produit une œuvre scientifique reconnue, tantôt occupent une position marginale, mais ils mettent en œuvre des stratégies relativement constantes. Or, tous ces traits apparaissent au XviIr siècle, comme le montrent les contributions rassemblées dans un numéro de la Revue d'histoire des sciences (vol. 44, 3/4, 1991) sur la « Diffusion des sciences au XviII ${ }^{\mathrm{e}}$ siècle $"$.

Par-delà l'explosion quantitative des années 1850 , par-delà même les déclarations d'intentions du début du $\mathrm{xIX}^{\mathrm{e}}$ siècle, le premier temps de

10. Claude Blanckaert, « La médecine philosophique de F. V. Raspail. Stratégies d'une "science populaire" ", in Raspail et la vulgarisation médicale, dir. Jacques POIRIER et Claude Langlors, Paris, Vrin, 1988, p. 129-198. Pour une approche comparatiste de la « science populaire ", cf. Susan Sheets-Penyson, « Popular Science Periodicals in Paris and London: The Emergence of a Low Scientific Culture, 1820-1875 ", Annals of Science, vol. 42, 1985, p. 549-572.

11. Mathias-Jacob Schleiden, La Plante et sa vie. Leçons populaires de botanique à l'usage des gens du monde, trad. de l'allemand par M. SCHEIDWEILER et P. ROYER, Paris, Schulz et Thuilié, 1859.

12. Cf. Claude Langlois et François Laplanche, dir., La Science catholique. L'Encyclopédie théologique de Migne (1844-1873) entre apologétique et vulgarisation, Paris, Cerf, 1992. 
cette histoire se situe - comme pour les publications scientifiques savantes - aux alentours de l'année 1700 ; c'est là qu'il faut chercher la naissance de la vulgarisation scientifique en tant que genre littéraire. Cette période initiale est à prendre en compte non pas seulement parce que des œuvres comme les Entretiens sur la pluralité des mondes de Fontenelle (1686), ou le Spectacle de la nature de l'abbé Pluche (1732) sont restées dans la mémoire du genre, mais plus fondamentalement parce que c'est à ce moment qu'est formulée la distinction entre deux types de littérature scientifique : l'une destinée aux savants et l'autre aux curieux.

\section{LA SCIENCE AU MIROIR DE LA VULGARISATION}

Distinction ne veut pas nécessairement dire coupure et, à cet égard, certains textes scientifiques majeurs sont nés de l'effort pour surmonter cette division du travail entre ceux qui font des découvertes et ceux qui les narrent au public. Buffon est l'exemple même d'un auteur animé par ce souci ${ }^{13}$. Il n'est pas le seul. D'autres noms reviennent constamment : ceux de Humboldt et d'Arago, qui apparaissent selon l'heureuse formule de Bruno Béguet, comme les "pères tutélaires de la vulgarisation française ", mais d'autres encore, plus inattendus, comme ceux de Laplace ou de Cuvier ${ }^{14}$. Ainsi le zoologiste Armand de Quatrefages, lorsqu'il publie en 1854, sous le titre de Souvenirs d'un naturaliste, un recueil d'articles parus dans la Revue des Deux Mondes, se justifie en invoquant ses prédécesseurs.

« Si quelque esprit trop sévère me reprochait d'avoir dépensé à cette œuvre de vulgarisation un temps que j'aurais pu consacrer à des recherches nouvelles, je ne lui répondrais que par quelques noms propres. Laplace a écrit son Exposition du système du monde; Cuvier, son Discours sur les révolutions du globe; Arago, ses Notices, M. Fourens, ses Etudes et ses Histoires; M. de Humboldt, ses Tableaux de la nature et son Cosmos " 1 .

13. Cf. Jacques Roger, Buffon. Un philosophe au Jardin du roi, Paris, Fayard, 1989. L'œuvre de Buffon était d'autant plus présente à l'esprit de ceux et de celles qui ont contribué à ce recueil, qu'elle est associée à leurs yeux à une absence bien douloureuse. Sans la mort qui l'a frappé quelques semaines plus tôt, c'est en effet à Jacques Roger qu'il revenait de présider la journée de travail où les contributions de ce recueil ont été présentées et discutées. Cette journée, qui a eu lieu à la cité des Sciences et de l'Industrie, le 29 mai 1990, avait été organisée par le Centre de recherche en histoire des sciences et des techniques, et animée par Jorge Martinez et moi-même.

14. B. BEGUET, op. cit. supra n. 5, p. 23.

15. A[rmand] de Quatrefages, Souvenirs d'un naturaliste, Paris, Masson, 1854, t. 1, p. xv. 
Quatrefages ne se contente d'ailleurs pas de se placer sous ces illustres patronages; toute sa Préface, dont ces lignes ne constituent que le point d'orgue, pourrait faire figure de manifeste. Tout d'abord, il affirme sans ambages le caractère de plaidoyer pour la zoologie que revêt son ouvrage. Pour cela, il évoque quelques-unes de ses applications pratiques, puis insiste sur ses mérites proprement intellectuels. En effet, la « science des créations vivantes » est " propre à agrandir notre esprit en même temps qu'à ramener notre esprit vers Celui qui a tout créé ${ }^{16}$. Par ailleurs, l'habitude d' " observer », de " classer » et de " coordonner des masses de faits précis et d'idées reposant sur des choses, de manière à en saisir les rapports vrais et les conséquences les plus générales ", s'avère aussi formatrice pour l'esprit que les mathématiques ou les sciences expérimentales ${ }^{17}$. Après avoir ainsi exposé dans quelle intention il publie ce livre, l'auteur en vient à justifier la manière dont il l'a rédigé. Les articles qui composent ce recueil étaient parus initialement dans la Revue des Deux Mondes. Ils s'adressaient donc, dit Quatrefages, à un public « instruit, intelligent, mais peu familier avec les sciences naturelles $"{ }^{18}$. De cette définition de son public découle une stratégie bien précise :

« Presque partout j'ai laissé de côté les détails par trop techniques, et traité à peu près exclusivement des questions générales. Souvent j'ai cherché à imiter le médecin qui enveloppe de miel le médicament dont la saveur répugnerait au malade, et de là les détails descriptifs ou historiques qui accompagnent presque tous les chapitres de cet ouvrage $" 19$.

Mais qu'on ne s'y méprenne point, l'auteur tient à marquer que cette stratégie de séduction n'a déterminé que la forme et n'a jamais touché le fond lui-même :

« Là j’ai voulu être zoologiste aussi rigoureux que dans un travail rédigé pour mes confrères. Les faits dont j'ai parlé dans ces Souvenirs sont ceux qu'on retrouverait soit dans mes Mémoires, soit dans les œuvres scientifiques les plus sérieuses ; les idées que j'y ai développées sont celles que j'ai professées dans tous mes travaux $\gg{ }^{20}$.

16. Ibid., p. $x$.

17. Ibid., p. xII. Craignant peut-être qu'on voie dans cette conception théologique une marque de conformisme vis-à-vis du cléricalisme triomphant au début du Second Empire, Quatrefages précise en note : " Je tiens à rappeler que les articles auxquels je fais ici allusion ont tous été écrits il y a huit ou dix ans. "

18. Ibid., p. xii-xiii.

19. Ibid., p. xiii.

20. Ibid., p. xiv. 
Et Quatrefages n'hésite pas à conclure : "Sous ce rapport ce livre aurait pu être intitulé : Essai de zoologie et de physiologie générale. "Son propos révèle combien était perméable la frontière qui séparait la vulgarisation de la publication scientifique.

Dans les années 1970, les analyses sur la fonction sociale de la vulgarisation ont surtout mis l'accent sur la cassure entre le discours des vulgarisateurs et le discours scientifique proprement dit $^{21}$. Des travaux plus récents, qui se sont attachés à une analyse sémiologique des deux discours, amènent à nuancer cette vision dichotomique. Ainsi, dans un article publié en 1987, Daniel Jacobi appuie son hypothèse d'une « continuité des pratiques de socio-diffusion du savoir au sein du champ scientifique " par une étude comparée de trois textes, écrits par le même chercheur sur le même sujet : une publication de recherche, un article destiné aux scientifiques non spécialistes et un article visant le grand public ${ }^{22}$. Dans les trois articles, on trouve des métaphores fondées sur des analogies dont il est difficile de décider s'il s'agit d'analogies à usage purement pédagogique ou à finalité heuristique. L'analyse porte sur une science, la biologie, qui fait - comme le note Jacobi - un large usage de l'analogie. On peut ajouter que le texte de vulgarisation choisi s'adresse à un public attentif et intéressé. En choisissant d'autres disciplines et d'autres supports on trouverait sans doute des discontinuités plus marquées.

Aucun des textes rassemblés ici n'entend trancher définitivement cette question de la cassure et de la continuité entre texte savant et texte de vulgarisation; tous, en revanche, éclairent cet entre-deux dans lequel s'échangent images et concepts. Dans $œ$ secteur des sciences naturelles, des images fantasmatiques se rencontrent parfois au détour des textes les plus savants ; des textes destinés au grand public peuvent laisser pressentir des voies nouvelles de recherche. La limite entre l'imaginaire et le vérifiable existe là comme ailleurs, mais là plus qu'ailleurs elle est sinueuse et incertaine, traverse les textes en eux-mêmes et frange les concepts les plus sûrs d'un halo de subjectivité.

Jean-Marc Drouin, Paris, C.R.H.S.T., Cité des sciences et de l'industrie.

21. Cf., en part., Philippe Roqueplo, Le Partage du savoir, Paris, Seuil, 1974, et Baudouin JURDANT, " La vulgarisation scientifique ", La Recherche, vol. 5, 53, 1975, p. 141-155.

22. Il s'agit de trois articles de Jacques Le Magnen sur la neurophysiologie de la faim, parus l'un dans les Archives italiennes de biologie, le deuxième dans La Recherche, le troisième dans Science et Vie ; cf. Daniel JACOBI, «Quelques formes du savoir savant dans les discours de vulgarisation scientifique ", Aster, 4, 1987, p. 91-117. 